\title{
Monostotic Type of Fibrous Dysplasia of Maxilla
}

\author{
Suresh Kumar Dhonvan ${ }^{1}$, Pavan Kumar B²,
} Srinivas Gadipelly³, Haripriya Chari ${ }^{4}$

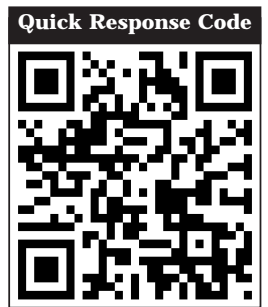

doi: $10.5866 / 2016.8 .10263$

${ }^{1}$ Post Graduate Student

${ }^{2}$ Professor \& HOD

3Professor,

${ }^{4}$ Senior Lecturer,

Department of Oral \& Maxillofacial Surgery,

Kamineni Institute of Dental Sciences,

Narketpally- 508254.

\section{Article Info:}

Received: October 11, 2016

Review Completed: November 09, 2016

Accepted: December 10, 2016

Available Online: December, 2016 (www.nacd.in)

(c) NAD, 2016 - All rights reserved

\section{Email for correspondence:}

dsureshomfs@gmail.com

\begin{abstract}
:
Fibrous Dysplasia is a non-neoplastic devel opmental disease of bone, which is manifested as a defect in osteoblastic differentiation and maturation. It appears in three forms namely monostotic (single bone invol vement), polyostotic (multiple bone involvement) and craniofacial forms. Of the craniofacial bones, Maxilla is affected more commonly than mandible. The present paper discusses a case of monostotic fibrous dysplasia of maxilla. It was diagnosed based on clinical, radiological and histopathological investigations. The osseous recontouring was done and he is under followup without any recurrence.
\end{abstract}

Key words: Fibrous dysplasia, monostotic type.

\section{INTRODUCTION}

Fibrous dysplasia is a bone development anomaly characterized by hamartoma proliferation of fibrous tissue within the medullary bone, with secondary bony metaplasia, producing immature, newly formed and weakly calcified bone, without maturation of the osteoblast which appears radiolucent on radiographs, with the classically described ground-glass appearance. ${ }^{1}$
In 1937, McCune and Bruch first suggested that among all of the abnormalities of bone formation, this disorder should have its own place as a distinct clinical entity. The following year, Lichtenstein introduced the term "fibrous dysplasia". There are three main types of Fibrous Dysplasia: monostotic, polyostotic, and McCune Albright syndrome. The monostotic form is the most common, comprising $70 \%$ of cases. $25 \%$ of $\mathrm{F}$ ibrous Dysplasia involves two or more bones. ${ }^{2}$ These lesions may be localized to 
one region of the body or they may be disseminated, involving al most every bone. These lesions are more likely to continue to progress even after puberty, specially craniofacial lesions. ${ }^{3}$

Fibrous dysplasia is a devel opmental tumor like sporadic condition that results from a post zygotic mutation in GNAS1 (Guanine Nucleotidebinding Protein, á stimulating activity polypeptide 1) gene. GNAS1 gene codes for $G$ protein which stimulates CAMP production in affected tissue; which result in Endocrinal disturbances leading to precocious puberty, hyperthyroidism, growth hormone and cortisol over production Increased proliferation of melanocyte leading to cafe-au-lait spots. Aberrant activity during osteoblasts differentiation, which results in normal medullary bone to be replaced by fibrous tissue and appears radiolucent on the radiograph. ${ }^{4}$

\section{CASE REPORT}

A 18 year old male patient reported to the department of oral and maxillofacial surgery with a chief complaint of swelling on the upper right back tooth .region from 6 years, which showed gradual increase in size without associated pain. On extra oral examination there was no noticible change in the facial symmetry (Figure 1). On intra oral examination there was a solitary swelling with illdefined borders of size $5 \times 4$ centimeter was noted on the right buccal gingiva extending from mesial aspect of 14 to distal aspect of 17 (Figure 2). There was rotation of second premolar which is not in contact with opposite tooth. On palpation the inspector findings were confirmed. Theswelling was hard in consistency, and was non reducible, non pulsatile and non compressible. It was firmly adherent to the underlying structures.

On radiographic examination, Orthopantomograph showed dense radiopaque area occupying entire right posterior maxillary jaw bone with ground glass appearance. Para nasal sinus view showed involvement of right maxillary sinus and reduction in its size (Figure 3 ). CT scan in coronal sections showed el evated right maxillary sinus floor and mixed radiolucent and radiopaque mass beneath it (Figure 4). Considering the features based on clinical and radiographic features a provisional diagnosis of Fibrous Dysplasia was made.

\section{Surgical Procedure}

Osseous recontouring was planned under general anesthesia. Left nasotracheal intubation was done. Crevicular incision along gingival sulcus was made extending from canine to the tuberosity with a vertical releasing incision at canine and full thickness mucoperiosteal flap was raised exposing the buccal cortical plate of the maxilla (Figure 5). Cuts around the lesion were marked using 703 straight fissure bur. Using osteotome a large part of buccal cortical plate was removed from canine to tuberosity region and bone recountouring was done with large flame shaped tungsten carbide bur. The excised specimen of size $5 \times 3$ centimeter was sent for histopathological evaluation (Figure 6). Wound closure was done in one layer using 3-0 vicryl sutures. Pressure dressing was given. Post-operative recovery was uneventful.

$\mathrm{H} \& \mathrm{E}$ decalcified section shows an admixture of irregularly shaped immature and mature bony trabeculae with osteoblastic rimming and there is presence of osteocytes within the lacunae. Trabeculae were interconnected in few areas and are seen interspersed within the fibrous connective tissue stroma (Figure 7).

\section{DISCUSSION}

Fibrous Dysplasia is commonly benign lesion in which irregularly distributed spicules of bone lie in cellular fibrous stroma. ${ }^{5}$ The lesion is believed to be hamartomatous develeopmental abnormality of bone of unknown etiology. ${ }^{6}$ However, there is some evidence that the etiology of Fibrous Dysplasia may be local infection or trauma. ${ }^{7}$

Fibro-osseous lesions are disorder of bone in which immature matrix of woven bone replaces matrix of normal bone. During the maturation of the lesion, the fibrous connective tissue is replaced with irregularly patterned trabecular bone. The monostotic form generally occurs during the second decade of life and is dormant by the third decade. 


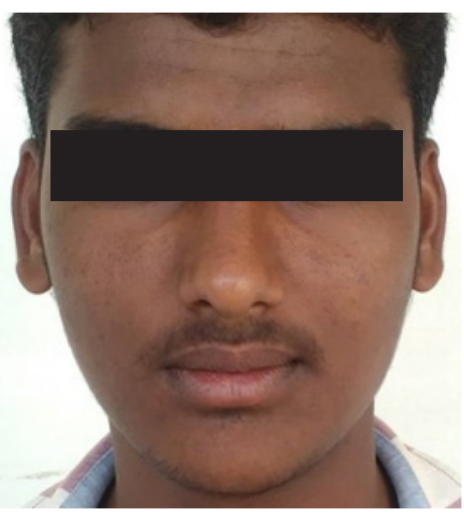

Figure 1: Clinical picture showing facial symmetry

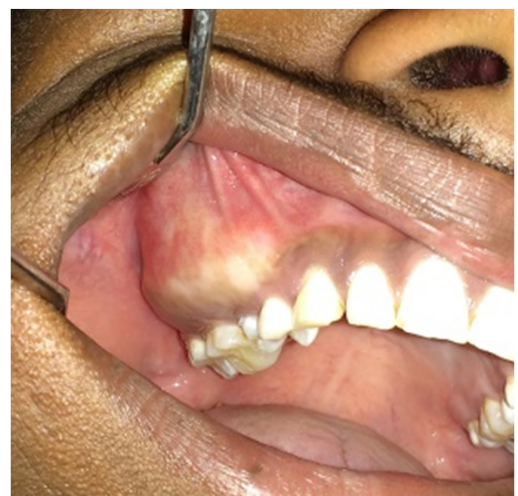

Figure 2: Iintra oral examination showing a solitary swelling with ill-defined borders of size

$5 \times 4$ centimeter noted on the right buccal gingiva extending from mesial aspect of 14 to distal aspect of 17

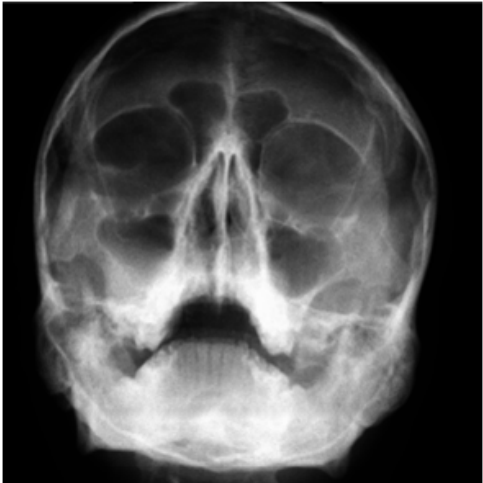

Figure 3: OPG showing dense radiopaque area occupying entire right posterior maxillary jaw bone with ground glass appearance.

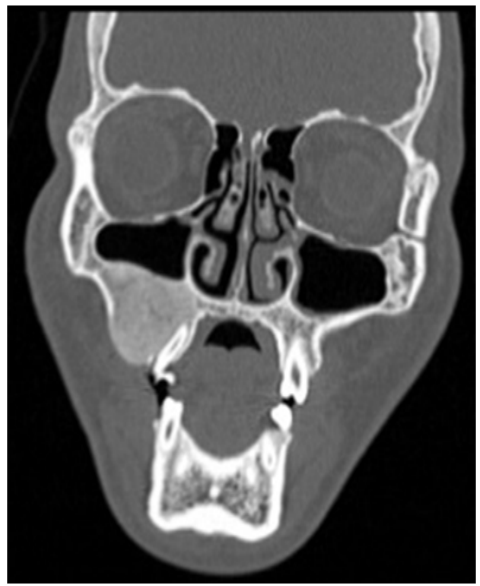

Figure 4: CT scan showing elevated right maxillary sinus floor along with mixed radiolucent and radiopaque mass beneath it

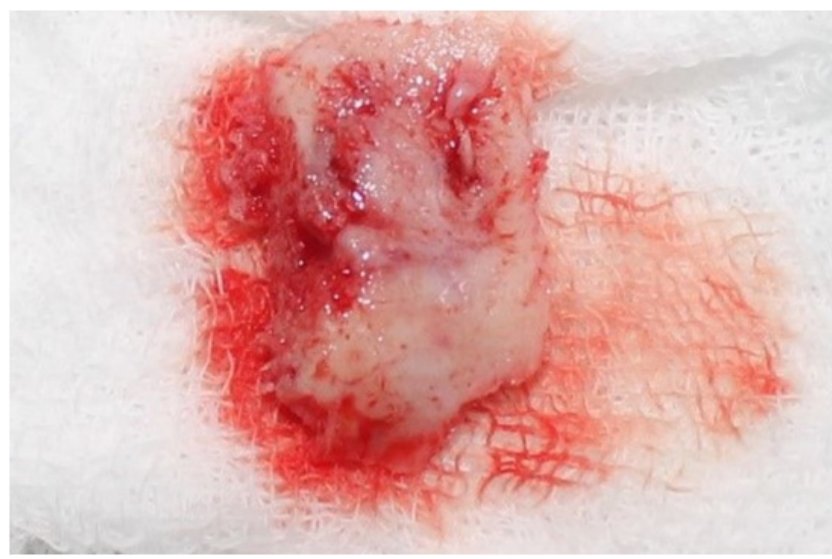

Figure 6: Excised mass of the lesion, sent for histopathological examination.

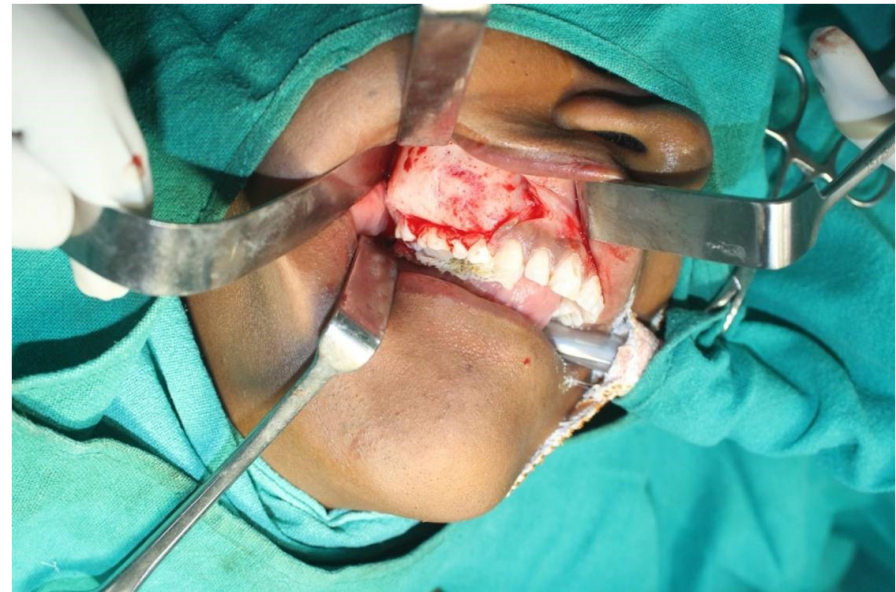

Figure 5: Surgical procedure exposing the lesion.

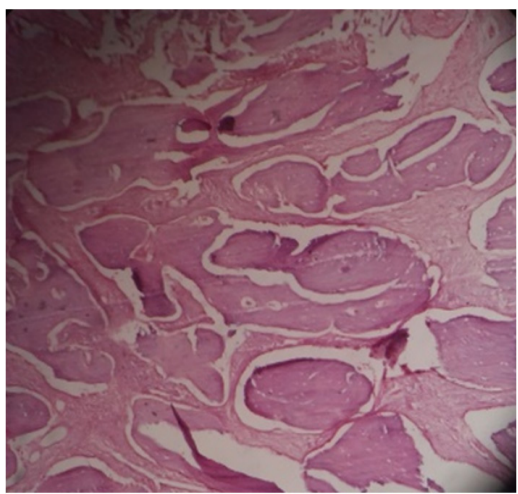

Figure 7: $\mathrm{H} \&$ E stained decal cified section shows an admixture of irregularly shaped immatureand mature bony trabeculae with osteoblastic rimming and there is presence of osteocytes within the lacunae. Trabeculae were interconnected in few areas and are seen interspersed within the fibrous connective tissue stroma 
The reactivation of the lesion can be seen due to hormonal changes, such as those seen in pregnancy. ${ }^{8}$

When maxilla is affected it may involve zygomatic and sphenoid bone. I nvolvement of frontal, sphenoid, naso-ethmoid and maxillary bone may lead to nasal obstruction, sinus obstruction and sinusitis. Malignant changes with fibrous dysplasia include Osteosarcoma, Fibrosarcoma, Chondrosarcoma, Malignant fibrous histiocytoma and Ewings sarcoma. ${ }^{9}$ Treatment is primarily surgery. When the only tooth bearing area is involved conservative treatment is bone shaving. Use of calcitonin \& pamindronate is also reported for its treatment. ${ }^{10}$ Biopsy can be taken to rule out the lesion. Fibrous dysplasia usually get stabilized after puberty. ${ }^{9}$

\section{CONCLUSION}

Fibrous dysplasia is the most common fibro osseous lesion affecting single bone or multiple bones. It often occurs in younger individuals and grows tolarge size which can cause marked esthetic and functional disturbances. Treatment modalities differ because of benign nature and dormant status after completion of growth. Small lesions causing no cosmetic and functional disturbances are left for observation and larger lesions aretreated with bone recontouring and resection.

\section{REFERENCES}

1. Cholakova R, Kanasirska P, Kanasirski N, Chenchev I, Dinkova A. Fibrous dysplasia in the maxillomandibular region-Case report. J IMAB 2010; 6(4):10-3.
2. Edgerton MT, Persing J A, J ane J A. The surgical treatment of fibrous dysplasia. With emphasis on recent contributions from cranio-maxillo-facial surgery. Annals of surgery 1985; 202(4):459.

3. Park BY, Cheon YW, Kim YO, Pae NS, Lee WJ . Prognosis for craniofacial fibrous dysplasia after incomplete resection: age and serum alkaline phosphatase. Int J Oralmaxillofacial Surg 2010; 39(3):221-6.

4. Rajendran R. Shafer's textbook of oral pathology. Elsevier India; 2009.

5. Canýtezer G, Gunduz K, Ozden B, Kose HI. Monostotic fibrous dysplasia: A case report. Dentistry 2012; 3:2.

6. Ozek C, Gundogan H, Bilkay U, Tokat C, Gurler T, Songur E. Craniomaxill ofacial fibrous dysplasia.J Craniofacial Surg 2002; 13(3):382-9.

7. Arshad N, Kapala J T. Monostotic fibrous dysplasia in an eight year old male: report of case. ASDC journal of dentistry for children 1995; 62(2):145.

8. Kaur B, Sheikh S, Shambulingappa S. Fibro-osseous lesion of maxilla. Report of two cases in a family with review of literature.

9. Keskin M, Karabekmez FE, Ozkan BT, Tosun Z, Avunduk MC, Savaci N. Simultaneous occurrence of facial fibrous dysplasia and ameloblastoma. J Cranio-Maxillofacial Surg 2009; 37(2):102-5.

10. Yasuoka T, Takagi N, Hatakeyama D, Y okoyama K. Fibrous dysplasia in the maxilla: possible mechanism of bone remodeling by calcitonin treatment. Oral oncology 2003; 39(3):301-5.

\section{Gain quick access to our journal online View our journal at www.nacd.in}

